\title{
Non-linear kinetics of damage accumulation and anomalies in the elastic properties of metals at very high cycle loading
}

\author{
O.B. Naimark ${ }^{\dagger}$, M.V. Bannikov \\ †naimark@icmm.ru
}

Institute of Continuous Media Mechanics Ural Branch of RAS, Perm, Russia

\begin{abstract}
The paper describes current understanding of the mechanism of generation of cyclic irreversible shifts as structurally induced plastic shear strains that, are generally a part of the accumulated plastic strain, which lead to the formation of fatigue cracks. Statistical approach is proposed, which allowed one to determine the types of collective modes in ensembles of mesoscopic defects and establish their role in the mechanisms of low- and high-cycle fatigue fracture, scaling mechanisms during fracture. A system of constitutive equations for low- and high-cycle fatigue is presented. An attempt is made to describe the relation between the microscopic mechanisms of fatigue and model representation and consideration of damage stages on the basis of non-linear kinetics of defects accumulation during cyclic loading in the regimes of high- and giga-cycle fatigue. A technique for «in situ» determination of fatigue damage accumulation is presented based on an analysis of the nonlinearity of feedback signal in the closed system of ultrasonic fatigue testing set. In the ultra high cycle (gigacycle) fatigue regime anomalies of elastic properties of the material are manifesting that leads to an effect of non-linearity of the oscillation amplitude. This effect increases with the initiation and growth of fatigue cracks. The method has been applied to samples with different pore sizes and at different levels of average stress in order to determine the moment of initiation and growth of fatigue cracks in the regimes of high- and gigacycle fatigue. It is shown that the technique can be used for an early detection of fatigue cracks both on the surface and within the material during cyclic loading in ultrasonic regimes.
\end{abstract}

Keywords: cyclic loading, irreversible deformation, scaling, collective modes of defects, non-linear kinetics of defects accumulation, high cycle fatigue, very high cycle fatigue.

\section{Нелинейная кинетика развития поврежденности и аномалии упругих свойств металлов при гигацикловом нагружении}

\author{
О.Б. Наймарк ${ }^{\dagger}$ М.В. Банников \\ †naimark@icmm.ru \\ Институт механики сплошных сред УрО РАН, ул. Ак. Королева 1, 614013, Пермь, Россия
}

В работе описаны современные представления о механизмах зарождения необратимых циклических сдвигов как структурно индуцированных пластических сдвиговых деформаций, являющихся в общем случае частью накапливаемой пластической деформации, которые приводят к образованию усталостных трещин. Приводится статистический подход, который позволил определить типы коллективных мод в ансамблях мезоскопических дефектов, установить их роль в механизмах мало- и многоциклового усталостного разрушения, механизмов скейлинга при разрушении. Приведена система определяющих уравнений для мало- и многоцикловой усталости. Представлена попытка описания связи микроскопических механизмов усталости с модельными представлениями и рассмотрением стадийности развития поврежденности на основе нелинейной кинетики накопления дефектов в процессе циклического нагружения в режимах много- и гигацикловой усталости. Приведена методика «in situ» определения накопления необратимых усталостных повреждений, основанная на анализе нелинейных проявлений сигнала обратной связи в замкнутой системе ультразвуковой усталостной установки. В режиме сверхмногоцикловой (гигацикловой) усталости проявляются аномалии упругих свойств материала, что приводит к эффекту нелинейности в амплитуде колебаний. Данный эффект возрастает с инициированием и ростом усталостной трещины. Данная методика была применена к образцам с различными размерами пор и при различном уровне среднего напряжения для определения момента инициирования и роста усталостной трещины в режимах много- и гигацикловой усталости. Показано, что методика применима для раннего обнаружения усталостных трещин как на поверхности, так и внутри материала при циклическом нагружении в ультразвуковом режиме.

Ключевые слова: Циклическое нагружение, необратимые деформации, скейлинг, коллективные моды дефектов, нелинейная кинетика накопления дефектов, многоцикловая усталость, гигацикловая усталость. 


\section{1. Введение}

В настоящее время после нескольких десятилетий исследований становится ясно, что усталостная поврежденность является результатом множественных многомасштабных деформаций, которые приводят к необратимым микроструктурным и топологическим изменениям, определяющим критические состояния поврежденности и переход к разрушению. Общие знания о механизмах циклической деформации и микроструктурных изменениях, приводящих к усталостному разрушению, позволяют связать эти критические состояния с развитием ансамбля микротрещин, зарождающихся в локализованных сдвигах.

В последние годы резко возрос интерес к фундаментальным проблемам усталости в связи с возможностью достижения усталостного ресурса, соответствующего так называемой сверхмногоцикловой (гигацикловой) усталости [1-10]. Результаты этих исследований поставили, в частности, вопрос о существовании предела усталости при переходе от много- к гигацикловым условиям нагружения.

Развитие повреждений при усталости традиционно связывается с микропластическими деформациями [1-2], которые формируются в условиях циклического нагружения, инициирующего различные микроструктурные механизмы, контролирующие долговечность и зависящие от природы и исходной структуры материала. Для пластичных металлов наблюдается специальный вид усталостной (циклической) локализации деформации (persistent slip bands - PSB), развитие которой традиционно ведет к инициированию трещин в приповерхностной зоне. Вторым распространенным случаем, например, при усталостном разрушении высокопрочных сталей, является зарождение повреждений (микротрещин), в окрестности включений при низких амплитудах нагрузки, соответствующих сверхмногоцикловой усталости (СМУ).

\section{1. Зарождение и интенсивность циклических необратимых сдвигов}

Величина необратимых сдвигов р определяется в [1] как микроструктурно-индуцированная пластическая сдвиговая деформация, являющаяся в общем случае частью накапливаемой пластической деформаций $[2,8]$. К микроскопическим механизмам, инициирующим циклические необратимые сдвиги, традиционно относят поперечные сдвиги в ансамблях винтовых дислокаций, сдвиговую асимметричность сдвигов, которые приводят к шероховатости поверхности образцов.

Поврежденности, инициируемые необратимыми сдвигами, не обязательно появляются на поверхности; они могут также формироваться в объеме материала или приповерхностных слоях. Переходы от сверхмногоцикловой усталости (низкая амплитуда нагрузки) к малоцикловой (высокая амплитуда) соответствуют изменению $p$ в диапазоне от $0<p<1$.

В $[2,8]$ отмечается, что определение вклада необратимого циклического сдвига представляет существенные экспериментальные трудности, и необходимы дополнительная экспериментальная информация и адекватные модельные представления, которые позволят провести комплексный количественный анализ. Так, приведенная в [1] оценка величины необратимого циклического сдвига для меди в условиях СМУ для числа циклов $10^{10}$ дает значение $p \sim 10^{-5}$, что составляет $10 \%$ от значения необратимого циклического сдвига при малоцикловой усталости.

\section{2. Определение усталостной долговечности по данным необратимого циклического сдвига}

В силу разнообразия механизмов и вклада в стадийность разрушения при усталости различных материалов, представляется проблематичным использование количественной величины параметра циклического сдвига для описания усталостной долговечности. Различие между стадиями зарождения и распространения трещин не является однозначным [7], что связано в большей степени с разрешением используемой экспериментальной техники. С учетом этого, выделяются следующие качественные признаки характерных стадий. Стадия инициирования, которая включает все механизмы формирования трещины до размера, с которого трещину можно рассматривать как дефект, распространение которого может описываться подходами механики разрушения (механики трещин). Это определение, предложенное в [8], соответствует структурной интерпретации как переход от состояния, контролируемого развитием системы микросдвигов, микротрещин при циклической пластической деформации, к стадии распространения трещины, контролируемой соотношениями механики разрушения.

В соответствии с данной интерпретацией стадийности, в $[9,10]$ был развит подход, получивший название «критической диаграммы поврежденности Френча» (the French critical damage curve), в основу которого было положено предположение о существовании на S-N диаграмме предела усталости.

Критическая кривая была получена в условиях двухстадийного эксперимента, соответствующего определению, данному в [9] для характерных стадий усталостного разрушения. Следуя данному определению, вводится количество циклов, необходимых для инициирования трещины «критической длины» («нераспространяющаяся трещина» в соответствии с диаграммой Kitagava-Takahashi [10]). Характерная длина таких формирующихся микротрещин была определена в $[9,10]$ в диапазоне до нескольких десятков микрон. Количество циклов, которые разделяют кривую Френча от S-N кривой при заданном напряжении, определяет временной период распространения трещины.

Важными при переходе от большеамплитудных режимов усталости (малоцикловая усталость) к малоамплитудным (много- и сверхмногоцикловая усталость) являются закономерности стадийности развития усталостного разрушения и рост продолжительности стадии 
развития поврежденности и формирования трещины по сравнению со стадией её распространения.

\section{3. Особенности зарождения трещин при сверхмногоцикловой усталости}

Особенностью усталостного разрушения при сверхмногоцикловой усталости является инициирование трещины в объеме материала, когда очаг разрушения имеет вид так называемого «рыбьего глаза» - «fish-еуе». При этом отмечаются два характерных случая, соответствующих формированию зоны «fish-еуе» в окрестности включения в высокопрочных материалах и в зоне интенсивных микропластических локализованных сдвигов в пластичных материалах.

В соответствии с рассматриваемыми случаями различают два типа материалов, которые обнаруживают многостадийные усталостные диаграммы в «CoffinManson» переменных $\left(\Delta \varepsilon_{\mathrm{pl}} / 2-\right.$ амплитуда пластической деформации; $N_{f}-$ критическое число циклов): пластичные как правило, имеющие ГЦК решетку, монофазные материалы (тип I) и высокопрочные стали, содержащие неметаллические включения (тип II).

\section{3. Система определяющих уравнений для мало- и многоцикловой усталости}

\section{1. Кинематические и силовые соотночения}

Кинематические соотношения для случая мало- и многоцикловой усталости в скоростях деформации могут быть представлены сє்(1)роответственно в виде:

$$
\begin{gathered}
\dot{\varepsilon}=\dot{\varepsilon}_{e}+\dot{\varepsilon}_{p}+\dot{p}, \\
\dot{\varepsilon}=\dot{\varepsilon}_{e},
\end{gathered}
$$

где $\dot{\varepsilon}$ - полная скорость деформации; $\dot{\varepsilon}_{e}-$ скорость упругой деформации; $\dot{\varepsilon}_{p}$-скорость пластической деформации, обусловленная движением дефектов по «механизму Орована», для которого получается простой механический аналог; $\dot{p}$-скорость деформации, обусловленная «геометрическим» вкладом дефектов. В условиях развитых пластических деформаций, наблюдаемых при малоцикловой усталости, «геометрический» вклад дефектов может быть незначительным и, как следствие, соотношение (1) представимо в виде $\dot{\varepsilon}=\dot{\varepsilon}_{e}+\dot{\varepsilon}_{p}$. Для много- и сверхмногоцикловой усталости соотношение (2) отражает механическую обратимость деформации в условиях развития повреждений, инициирующих микропластические сдвиги.

Представление полных напряжений $\sigma$ для всех рассматриваемых типов нагружений включает упругую (обратимую) и необратимую (диссипативную) компоненту:

$$
\sigma=\sigma_{e}+\sigma_{p}
$$

где $\sigma_{e}$ - «упругая» компонента, $\sigma_{p}$ - компонента, соответствующая диссипативным механизмам: микропластическим деформациям при много- и сверхмногоцикловой усталости, развитым пластическим деформациям при малоцикловой усталости.

\section{2. Основные термодинамические соотночения}

Статистико-термодинамическое описание коллективного поведения ансамблей мезоскопических дефектов (микротрещин, микросдвигов), развитое в [11], позволило предложить феноменологическое представление неравновесной свободной энергии материала с дефектами $F(\varepsilon, p, \delta)$, установленной в ходе статистикотермодинамического описания материала с дефектами.

$$
\begin{aligned}
& F=F_{e}\left(\varepsilon_{e}\right)+1 / 2 A\left(1-\delta / \delta_{*}\right) p_{k}^{2}-1 / 4 \beta_{k}^{4}+ \\
& +1 / 6 C\left(1-\delta / \delta_{c}\right) p_{k}^{6}-D \sigma_{k} p_{k}+\chi\left(\nabla_{l} p_{k}\right)^{2},
\end{aligned}
$$

где $A, B, C, D$ - параметры материала, $\chi$ - коэффициент «нелокальности», связанный, например, с размером зерен; $F_{e}$ - «упругая» компонента, остальные слагаемые аппроксимируют (по аналогии с разложением ГинзбургаЛандау) результаты статистического описания в рамках двух дополнительных переменных, характеризующих поведение материала с дефектами: тензор плотности дефектов $p_{i k}$ (совпадающий по смыслу с «геометрической деформацией», индуцированной дефектами и, как следствие, появляющейся в кинематическом соотношении (1), где $p \sim p_{i k}$ ) и параметр структурного скейлинга $\delta$. Существование последнего было установлено в рамках статистического описания, но ранее этот параметр был обнаружен при анализе экспериментальных данных в [11] как факт «статистической автомодельности» в распределении дефектов на различных стадиях деформирования, в том числе усталости. Параметр структурного скейлинга представляет собой отношение двух масштабов: расстояние между дефектами $L_{s c}=(\mathrm{n})^{-1 / 3}$ и размер дефекта $l_{s c}$ : $\delta \sim\left(L_{s c} / l_{s c}\right)^{3}$. Являясь переменной состояния, $\delta$ определяет «текущую восприимчивость» материала к росту плотности дефектов. Принципиально важно, что статистическое описание установило существование двух критических значений для параметра структурного скейлинга $\delta_{c}=1$ и $\delta_{*} \sim 1.3$ и соответствующие «диапазоны» структурной восприимчивости материала к росту плотности дефектов: квази-хрупкий $\left(\delta<\delta_{c} \approx 1\right)$, «вязкий» $\left(\delta_{c}<\delta<\delta_{*}\right)$ и субмикрокристаллический $\left(\delta>\delta_{*}\right)$. Критические значения $\delta_{c}$ и $\delta_{*}$ являются аналогами критических температур в теории Гинзбурга-Ландау. Переходы через критические значения $\delta$ в ходе накопления дефектов приводят к формированию качественно различных коллективных мод дефектов (автомодельных решений для уравнений кинетики поврежденности для $p_{i k}$ и $\delta$ ) с зарождением и кинетикой которых будет связана стадийность развития поврежденности: формирование областей локализованного сдвига и очагов разрушения.

Система определяющих соотношений следует из соотношений (1)-(4) и выражения для диссипативной функции D среды с дефектами [14]. Диссипативная функция имеет качественно-различный вид (температурные слагаемые опущены) для случая много- и малоцикловой усталости в зависимости от вида кинематического соотношения (1) и представления полных напряжений (2).

Малоцикловая усталость:

$$
D=\sigma_{e}: \dot{\varepsilon}_{p}-\frac{\partial F}{\partial p}: \dot{p}-\frac{\partial F}{\partial \delta}: \dot{\delta}>0
$$


Многоцикловая усталость:

$$
D=\sigma_{p}: \dot{\varepsilon}-\frac{\partial F}{\partial p}: \dot{p}-\frac{\partial F}{\partial \delta}: \dot{\delta}>0 .
$$

Определяющие соотношения следуют из условий знакоопределенности диссипативной функции с учетом представления для неравновесной свободной энергии и соотношений Онсагера.

$$
\begin{aligned}
& \sigma_{p}=\Gamma_{1}: \dot{\varepsilon}-\Gamma_{2}: \dot{p}, \\
& -\frac{\partial F}{\partial p}=\Gamma_{2}: \dot{\varepsilon}+\Gamma_{3}: \dot{p}, \\
& \dot{\delta}=-\Gamma_{4} \frac{\partial F}{\partial \delta},
\end{aligned}
$$

где $\Gamma_{i}(\mathrm{i}=1, \ldots .4)$ - кинетические коэффициенты.

С учетом представления полных напряжений возможна замена напряжения $\sigma_{p}$ на макроскопически наблюдаемые переменные $\sigma_{p}=\sigma-\sigma_{e}$ где $\sigma_{e}$, например, для чистого сдвига, $\sigma_{e}=G \varepsilon$, где $G$ - модуль сдвига. Используя эту замену, систему определяющих соотношений можно привести к виду:

$$
\begin{aligned}
& \dot{\varepsilon}=-\frac{\varepsilon}{\tau_{\varepsilon}}+\frac{\sigma}{\Gamma_{1}}-\frac{\Gamma_{2}}{\Gamma_{1}} \dot{p}, \\
& \dot{p}=\frac{\Gamma_{2}}{\Gamma_{3}} \dot{\varepsilon}-\frac{1}{\Gamma_{3}} \frac{\partial F}{\partial p}, \\
& \dot{\delta}=-\Gamma_{4} \frac{\partial F}{\partial \delta} .
\end{aligned}
$$

Первое уравнение в (8) описывает процесс релаксации деформаций с характерным временем $\tau_{\varepsilon}=\Gamma_{1} / G$ - временем ретардации при действии макроскопического напряжения $\sigma, \Gamma_{1}-$-коэффициент «микропластической» вязкости. Вклад в полную скорость деформации дают также дефекты через «перекрестный» член $\Gamma_{2} / \Gamma_{1} \dot{p}$, обеспечивая влияние на релаксационные процессы (включая гистерезисные вследствие нелинейности неравновесного потенциала) кинетики накопления дефектов в соответствии со вторым уравнением в (8).

\section{3. Стадийность развития поврежденности. Коллективные моды дефектов}

Кинетическое уравнение для параметра поврежденности имеет различные групповые свойства в соответствующих диапазонах параметра структурного скейлинга $\delta$, определяемые видом нелинейности неравновесного потенциала $F$, включая тип метастабильности. При $\delta \rightarrow \delta$ * происходит вырождение решений, характерных для параболического уравнения с нелинейным источником, и последнее трансформируется в волновое решение автосолитонного типа $p(\zeta)=p(x-V t)$ для бистабильного потенциала. Этот переход сопровождается резким изменением коллективной ориентационной моды дефектов и расходимостью внутреннего масштаба по регулярному сценарию. Амплитуда волны, её ширина и скорость волнового фронта определяются параметрами метастабильного перехода

$$
p=\frac{1}{2} p_{a}\left[1-\tanh \left(\zeta l^{-1}\right)\right] \quad l=\frac{4}{p_{a}}\left(2 \frac{\chi}{A}\right)^{1 / 2}
$$

$$
V=\chi A\left(p_{a}-p_{m}\right) / 2 \zeta^{2},
$$

где $\left(p_{a}-p_{m}\right)$ - скачок величины $p$ в ходе метастабильного перехода. Формирование пространственно локализованных волновых структур, фронты которых соответствуют «ориентационному переходу» в ансамблях дефектов, позволяют связать кинетику формирования этих структур с механизмами пластической релаксации (локализацией пластической деформации).

Переход через точку бифуркации $\delta_{c}$ сопровождается очередным изменением групповых свойств уравнения (3), что приводит к появлению пространственно-временных структур качественно-нового типа, описывающих «взрывное» накопление дефектов (так называемые режимы с обострением $[12,13])$ при $t \rightarrow t$. на спектре пространственныхмасштабов $L_{H}=k L_{c}, k=1,2, \ldots, K$. Для данного типа уравнений, как показано в [13], развитая стадия кинетики $p>p_{C}$ при $t \rightarrow t$, описывается автомодельным решением

$$
p(x, t)=\phi(t) f(\zeta) \quad \zeta=\frac{x}{L_{c}}, \quad \phi(t)=\Phi_{0}\left(1-\frac{t}{t_{c}}\right)^{-m}
$$

где $m>0, \Phi_{0}>0$ - параметры, определяемые показателями нелинейности потенциала $(1) ; L_{c}$ и $t_{c}$ - параметры скейлинга. Функция $f(\zeta)$ соответствует начальному автомодельному профилю поврежденности. “Подчинение” кинетики роста дефектов режиму с обострением соответствует сценариям автомодельности второго рода с вырождением масштабов, сформированных коллективными автосолитонными модами. Формирование структур обострения обеспечивает наиболее эффективный канал диссипации, который может быть связан с зарождением очагов макроскопического разрушения (трещин) в твердых телах. Признаки критического поведения ансамблей дефектов при переходе через значения $\delta_{*}$ и $\delta_{c}$ позволяют идентифицировать стадийность поврежденности в соответствии с аномалиями поглощения механической энергии, наблюдаемыми в окрестности критических точек.

\section{4. Нелинейная кинетика развития поврежденности и феноменологические законь усталости}

В области метастабильности неравновесного потенциала $\delta_{c}<\delta<\delta_{*}$ автомодельные решения имеют природу автосолитонных волн, фронты которых соответствуют появлению коллективной ориентационной моды дефектов (и имеют соответствующие образы на поверхности разрушения). Коллективные моды автосолитонной природы выступают в роли коллективных переменных с относительно медленной динамикой по отношению к внешним временам нагружения и «подчиняют» себе стадию подготовки разрушения в вершине трещины. Кинетика изменения $\delta$ в соответствии с (9) в диапазоне $\delta_{c}<\delta<\delta_{*}$ «подчиняет» релаксацию напряжений в вершине трещины метастабильным (ориентационным) переходам с порядком близким к 4 [13]. 


\section{4. Нелинейные эффекты упругости и податливости в поврежденных образцах}

Оценка стадийности процесса усталостного разрушения по данным ультразвуковых испытаний вызывает большой интерес как эффективный метод неразрушающего контроля. В [14-16] обсуждаются методы измерения амплитуды второй и более высоких гармоник основной частоты, которые позволяют обнаружить и охарактеризовать усталостные повреждения в металлах, которые вызывают отклонения от гармонических ультразвуковых режимов. Метод основан на инициировании продольного конечно-амплитудного возмущения $A_{0}$ с частотой $\omega_{0}$ с одной стороны образца, тогда как другой его торец остается свободным. При достаточно больших значениях $A_{0}$ и отклонениях от линейного упругого закона колебания свободного торца образца будут содержать ряд гармонических составляющих: компоненты с амплитудой $A_{1}$ на основной частоте $\omega_{0}$, амплитудой $A_{2}$ второй гармоники частотой $2 \omega_{0}$, и так далее. Параметр нелинейности $\beta$ е определяется экспериментально путем измерения абсолютных амплитуд сигналов первой A1 и второй $A_{2}$ гармоник, соответствующих нелинейному закону упругости:

$$
\begin{aligned}
& \sigma=A_{2}^{e}\left(\frac{\partial u}{\partial a}\right)+\left(\frac{1}{2}\right) A_{3}^{e}\left(\frac{\partial u}{\partial a}\right)^{2}+\ldots= \\
& =A_{2}^{e}\left[\left(\frac{\partial u}{\partial a}\right)-\frac{1}{2} \beta^{e}\left(\frac{\partial u}{\partial a}\right)^{2}+\ldots\right]
\end{aligned}
$$

где $\sigma$ - нагрузка, $u$ - перемещение, $a$ - пространственная координата, $A_{2}^{e}$ и $A_{3}^{e}$ - упругие коэффициенты второго и третьего по- рядка соответственно. Введением коэффициента нелинейности $\beta^{e}=-\left(A_{3}^{e} / A_{2}^{e}\right)$ волновое уравнение может быть представлено в виде

$$
\frac{\partial^{2} u}{\partial t^{2}}=c^{2}\left[1-\beta^{e}\left(\frac{\partial u}{\partial a}\right)\right]\left(\frac{\partial^{2} u}{\partial a^{2}}\right)
$$

где $u$ - компонента вектора смещений в направлении $a, c$ - продольная скорость звука, $t$ - время. Его решение, учитывая, что возмущение торца $u=u_{1} \cos (\omega t)$, будет иметь вид:

$$
u=u_{0}+u_{1} \cos (\omega t)+u_{2} \sin 2(\omega t-k a)+\ldots,
$$

где $u_{2}=(1 / 8) k^{2} \beta^{e} u_{1}^{2} a, k=\omega_{0} / v-$ волновое число. Откуда можно выразить:

$$
\beta^{e}=8 u_{2} / k^{2} u_{1}^{2} a .
$$

При исследовании нелинейных явлений в режиме гигацикловой усталости с помощью измерения амплитуд основной и второй гармоник определяется относительный параметр:

$$
\beta_{\text {relative }}=\beta / \beta_{0},
$$

где $\beta_{0}$ относится к неповрежденному материалу. Увеличение $\beta_{\text {relative }}$ с ростом усталостных повреждений сообщалось в ряде работ [17-20]. Разработанные в $[14,17,20]$ экспериментальные установки позволяют оперативно наблюдать процесс усталостного нагружения с помощью лазерного виброметра и определять высшие гармоники в испытуемых образцах.

\section{5. Измерение коэффициента нелинейности}

Образцы из алюминиевых сплавов были испытаны в работах [17-19] с амплитудой напряжений 130, 150 и 160 МПа. Эти значения напряжений находятся в интервале 0,45-0,55 от предела упругости 289 МПа исследуемого материала, имеющего модуль Юнга 69 ГПа. Во время импульса длительностью 100 мс, записывался сигнал длиной 10 мс (с частотой записи 250 кГц, то есть - 2500 точек) с задержкой записи 35 мс. Изменения резонансной частоты в течение циклических испытаний фиксировались по пиковым значениям из амплитудного спектра измеряемого сигнала.

Для определения коэффициента нелинейности прологарифмируем обе части уравнения (15):

$$
\log (\beta)=\log (k)+\log \left(u_{2}\right)-2 \log \left(u_{1}\right)
$$

В работах [15,17-19] амплитуда колебаний измеряется в децибелах в масштабах $A_{1}=20 \log \left(a_{1}\right)$ и $A_{2}=20 \log \left(a_{2}\right)$, тогда соотношение (7) принимает вид:

$$
\begin{gathered}
20 \log (\beta)=20 \log (k)+20 \log \left(u_{2}\right)-40 \log \left(u_{1}\right), \\
20 \log (\beta)=K+A_{2}-2 A_{1} .
\end{gathered}
$$

Таким образом, относительный параметр нелинейности в (15) можно найти из формулы:

$$
20 \log \left(\beta / \beta_{0}\right)=\left(A_{2}-2 A_{1}\right)-\left(A_{2}-2 A_{1}\right)_{0}
$$

На частотном спектре измеряемых сигналов в начале испытаний амплитуда второй гармоники очень мала, но в процессе циклических испытаний амплитуда 2й гармоники начинает увеличиваться. По формуле (21) из данных сигналов вычисляется коэффициент нелинейности. На рис. 1 показаны зависимости коэффициента нелинейности $\beta_{\text {relative }}$ и его изменения за цикл $d \beta / \mathrm{dN}$ во время испытаний образцов 6061-Т6511 при амплитудах напряжения 130, 150 и 160 МПа. Усталостная долговечность при 130,150 и 160 составила $1.63 \times 10^{7}, 2.87 \times 10^{6}$ и $0.83 \times 10^{6}$ циклов соответственно.

Как можно заметить из графика, наибольшее изменение $d \beta / \mathrm{dN}$ наблюдается при амплитуде нагружения 160 МПа, затем в порядке уменьшения следуют значения при 150 и 130 МПа соответственно. Этот ожидаемый результат соответствует величине скорости роста трещины и показывает возможность использования метода для “in situ” регистрации и контроля скорости роста усталостной трещины в процессе испытаний.

$\mathrm{B}[17]$ отмечено, что изменение $\beta_{\text {relative }}$ происходит зна-

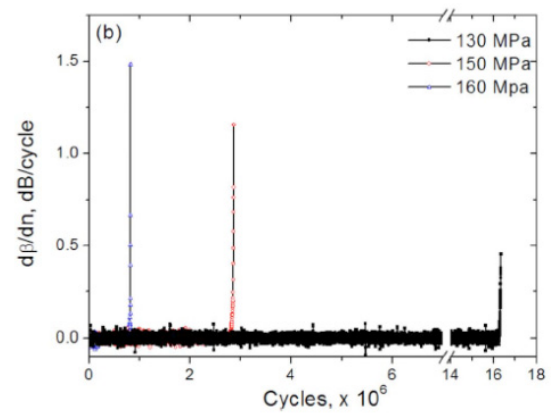

Рис. 1. Относительное изменение показателя нелинейности за цикл нагрузки.

Fig. 1. The relative change of the non-linearity coefficient for the load cycle. 
чительно раньше, чем изменение резонансной частоты (рис.2), что говорит о более высокой чувствительности метода.

Для подтверждения факта обнаружения внутренней трещины был проведен следующий эксперимент [19]: образец испытывался при амплитуде напряжений 90 МПа в течение $2.23 \times 10^{6}$ циклов. После обнаружения монотонного возрастания коэффициента нелинейности амплитуда была повышена до 150 МПа для окончатель-
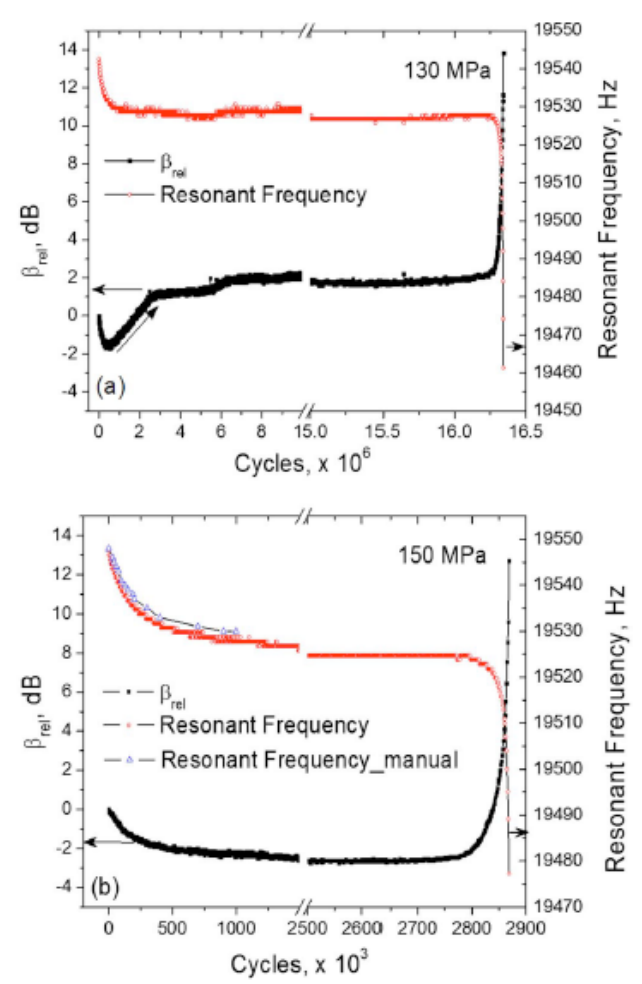

Pис. 2. Зависисмости коэффициента нелинейности и резонансной частоты от числа циклов.

Fig. 2. The dependencies of nonlinearity coefficient and resonant frequency on number of cycles.

ного разрушения образца. На поверхности разрушения были обнаружены области роста трещины при нагрузках 90 МПа и 150МПа с различным рельефом. Таким образом, данный метод применим для раннего обнаружения усталостной трещины даже при внутреннем ее зарождении.

\section{6. Заключение}

Показано, что усталостное разрушение связано с накоплением необратимых повреждений, вызванных нелинейными проявлениями циклического сдвига. Стадия инициирования усталостной трещины соответствует структурной интерпретации как переход от состояния, контролируемого развитием системы мезодефектов (микросдвигов, микротрещин) при циклической пластической деформации, к стадии распространения трещины, контролируемой соотношениями механики разрушения.
Приведены определяющие соотношения, описывающие нелинейную механику накопления дефектов в условиях циклической деформации.

Описана методика для определения накопления необратимых усталостных повреждений с помощью анализа нелинейных проявлений сигнала обратной связи в замкнутой системе ультразвуковой усталостной установки. В режиме сверхмногоцикловой (гигацикловой) усталости проявляются аномалии упругих свойств материала, что приводит к эффекту нелинейности, который возрастает с инициированием и ростом усталостной трещины. Стадийность развития поврежденности связывается с проявлениями аномалий поглощения энергии при формировании коллективных мод дефектов.

Приведены зависимости коэффициента нелинейности $\beta_{\text {relative }}$ от амплитуды нагружения в течение усталостной долговечности для алюминиевых сплавов с различным состоянием внутренних микропор. Показано, что Brelative значительно увеличивается при образовании усталостной трещины и его значение увеличивается с ростом амплитуды.

Показано, что чувствительность методики определения момента зарождения трещины по коэффициенту нелинейности превышает методику определения по резонансной частоте, что позволит в дальнейшем более подробно исследовать механизмы и характер возникновения внутреннего разрушения, называемого «fish-еуе».

Благодарность. Работа выполнена при поддержке гранта РНФ 14-19-01173.

Acknowledgements. This study was supported by the Russian Science Foundation, project No. 14-19-01173.

\section{Список литературы}

1. H. Mughrabi, // Int. J. of Fatigue 57, 2013, P. 2-8

2. Shyam A, Milligan W. Acta Mater 2005;53:835-44.

3. Risbet M, Feaugas X. Eng Fract Mech 2008;75:3511-9.

4. Bannikov M., Oborin V., Naimark O. and Froustey C. Tech. Phys. Lett. - 2011. - Vol. 37. - No. 3. - P. 241-243.

5. Bannikov M., Oborin V., Naimark O. Palin-Luc T. Tech. Phys. Lett. - 2010. - Vol. 36.

6. Weidner A, Man J, Tirschler W, Klapetek P, Blochwitz C, Polak J, et al. Mater Sci Eng A 2008;492:118-27.

7. Mughrabi H. Metall Mater Trans A 2009;40:1257-79.

8. Mughrabi H. Int J Fatigue 2006;28:1501-8.

9. Lukaš P. ASM handbook. Fatigue and fracture, v. 19. Materials Park, OH, USA: ASM International; 1996. p. 96-109.

10. French H.. Trans Am Soc Steel Treat 1933;21:899-946.

11. Naimark O.B. In: Advances in multifield theories of continua with substructure. Ed.: G.Capriz, P.Mariano.Boston, Birkhauser.- 2004. -P.75-114.

12. Kurdyumov S.P. Evolution and self-organization laws of complex systems // International Journal of Modern Physics.-1988.-V.1.-No.4.-P.299-327.

13. Belyaev V.V. Naimark O.B. Dokl. AS USSR 1990.- 312.No 2.- Рp.289-293. [Беляев В.В., Наймарк О.Б. Докл. 
AH CCCP.-1990.-T.312.-№ 2.-C.289-293.]

14. T. L. Grobstein, S. Sivashankaran, G. Welsch, N. Panigrahi, J. D. McGervey, and J. W. Blue, Mater. Sci, Eng. A 138, 191-203 (1991).

15. J. H. Cantrell, W. T. Yost. Int. J. of Fatigue 23 (2001) S487S490.

16. J.Y. Kim, L.J. Jacobs, J. Qu, J.W. Littles, J. Acoustic Soc. Am., 120.(1266), USA 2006.
17. A. Kumar, C.J. Torbet, J.W. Jones, T.M. Pollock. J. of App. Phys. 106, 024904 (2009).

18. A. Kumar, C.J. Torbet, T.M. Pollock, J.W. Jones. Acta Materialia 58 (2010) 2143-2154.

19. A. Kumar, R.R. Adharapurapu, J.W. Jones, T. M. Pollock. Scripta Materialia 64 (2011) 65-68

20. C. Froustey, T. Auzanneau, J.-L. Charles, and J.-L. Latailade, J. Phys. IV 10, PR9-565 (2000). 